\section{Mapeamento de internações hospitalares por problemas respiratórios e possíveis associações à exposição humana aos produtos da queima da palha de cana-de-açúcar no estado de São Paulo}

\section{Mapping of hospitalizations due to respiratory problems and possible associations to human exposure to burnt sugar-cane straw products in the state of São Paulo}

\section{Fábio Silva Lopes ${ }^{1}$}

Helena Ribeiro ${ }^{2}$

${ }^{1}$ Faculdade de Computação e Informática da Universidade Presbiteriana Mackenzie

${ }^{2}$ Departamento de Saúde Ambiental da Faculdade de Saúde Pública da Universidade de São Paulo

Correspondência: Fábio Silva Lopes. Faculdade de Computação e Informática da Universidade Presbiteriana Mackenzie. Rua da Consolação, 930 - Prédio 13 - Consolação - CEP - 01302-907 E-mail: flopes@mackenzie.com.br

\section{Resumo}

Introdução: O uso de Sistemas de Informações Geográficas em pesquisas na área de saúde pública é cada vez maior, devido às possibilidades de comparação de dados dispostos em tabelas e mapas através de sua sobreposição. Tal sobreposição possibilita uma nova leitura dos dados associados à dimensão do espaço. Os programas SIG são ideais para pesquisas em geografia da saúde. Em estudos ecológicos de causa/efeito, envolvendo variáveis ambientais e os efeitos deletérios à saúde humana, o geoprocessamento constitui um interessante recurso para gerar hipóteses para estudos mais aprofundados sobre o tema. Objetivo: Este estudo objetivou a construção de um SIG para verificar a possível correlação entre os produtos das queimadas de cana-de-açúcar e a incidência de problemas respiratórios em regiões afetadas, através das técnicas de geoprocessamento. Metodologia: Consistiu em organizar dados de queimadas e internações por afecções respiratórias do Estado de São Paulo, no período de 2000 a 2004, estruturando um SIG em duas escalas. Na primeira, foram criadas cartas temáticas de todo o Estado e identificadas áreas de interesse para estudos em escala local. A segunda consistiu na construção de SIG abrangendo a meso-região de Bauru, para observar a intersecção dos agravos à saúde em áreas de queimada. Resultados: Nos mapas elaborados e numa análise de correlações espaciais observou-se maior incidência de doenças respiratórias em regiões onde há prática de queimadas. O material produzido constitui um conjunto de dados que permite ao pesquisador verificar hipóteses para subsidiar estudos no âmbito da saúde ambiental.

Palavras-chave: Cana-de-açúcar. Geoprocessamento. SIG. Queimadas. Saúde Ambiental. Saúde Pública. Poluição do ar. São Paulo. Geografia da saúde. 
Abstract

Introduction: Geographic Information Systems (GIS) are being increasingly used in public health studies, as they allow comparing data organized in tables and maps through overlapping. Overlapping provides a new way of reading data associated with the spatial dimension. GIS programs are ideal for studies in health geography. Geoprocessing is an interesting resource for generating assumptions for more indepth studies on the theme in cause/effect ecological studies, comprising environmental variables and their deleterious effects to human health. Objective: The present study aimed to construct a GIS to verify the possible correlation between products of sugar-cane fires and the incidence of respiratory conditions in the affected regions, by using geoprocessing techniques. Methods: A two-scale GIS was structured from data on biomass fires and hospitalizations due to respiratory conditions in the State of São Paulo for the period from 2000 to 2004. For the first scale, theme maps were created for the entire State and interesting areas for local scale studies were identified. The second scale consisted of constructing a GIS encompassing the meso-region of Bauru, in order to verify the intersection of health hazards in biomass fire areas. Results: The maps and spatial correlation analysis showed a higher incidence of respiratory conditions in regions where biomass fires are used. The resulting material produced a set of data that allowed the investigator to verify assumptions that may support environmental health studies.

Keywords: Sugar cane. Geoprocessing. GIS. Biomass fire. Environmental Health. Public Health. Air pollution. São Paulo. Medical geography.

\section{Introdução}

O Brasil é o maior produtor de cana-deaçúcar (Saccharum sp) do mundo. Atualmente, 4,5 milhões de hectares do território nacional são utilizados para o plantio de cana, representando $1 \%$ das terras agricultáveis, ou o espaço equivalente a duas vezes o Estado do Piauí. A produção nacional é de 290 milhões de toneladas $/ \mathrm{ano}^{1}$. Da safra canavieira, $55 \%$ são destinados à produção de álcool e subprodutos, enquanto os demais $45 \%$ são destinados à produção de açúcar e subprodutos. Uma avaliação recente estimou em $12 \%$ a participação da agroindústria no PIB brasileiro².

No Estado de São Paulo, utiliza-se 2.531.000 hectares para o plantio da canade-açúcar, produzindo um montante de 230 milhões de toneladas/ano, que representa $60 \%$ da produção nacional. Neste cenário, $60 \%$ das áreas de plantio utilizam o processo de queima da palha antes da colheita.

Entre as idas e vindas dos vários ciclos agro-econômicos brasileiros, as áreas de plantio de cana-de-açúcar da atualidade concentraram-se principalmente nas regiões Centro-Sul e Nordeste do país. Atualmente, tais concentrações permitem dois períodos de safra por ano e a lavoura de cana-de-açúcar emprega um milhão de trabalhadores em uma atividade que ainda possui $80 \%$ do corte executado por processos manuais.

A prática de queimar a palha da canade-açúcar antes do corte causa uma modalidade de poluição do ar. Enquanto a emissão veicular de material particulado pode chegar a 62 toneladas/dia na Região Metropolitana de São Paulo, o material particulado proveniente da queima de palha, conhecido como "carvãozinho", pode chegar a 285 toneladas/ $\mathrm{dia}^{3}$.

No que tange à qualidade de vida das coletividades, a literatura indica uma série de conseqüências negativas que atinge a população, em razão do material particulado produzido pelas queimadas. Moradores que vivem em áreas afetadas rela- 
tam os seguintes problemas ${ }^{3}$ :

- Sujeira em casas, comércio e locais públicos;

- Aumento do consumo de água de abastecimento público para garantir a limpeza dos locais afetados com maior freqüência;

- Aumento dos acidentes em rodovias devido à falta de visibilidade;

- Problemas respiratórios, notadamente em crianças e idosos;

- Interrupção de serviços de energia elétrica por problemas causados em linhas de transmissão próximas a área da queimada;

- Desperdício de energia;

- Eliminação de animais silvestres, pássaros, etc.;

- Emissão de gases prejudiciais ao meio ambiente;

- Destruição da palha que não se incorpora ao solo.

Nos estudos epidemiológicos que envolvem poluição do ar e saúde, a determinação exata da exposição é um requisito importante para avaliação dos efeitos. Segundo Castro, Gouveia e EscamillaCejudo $^{4}$, a exposição a um poluente pode ser definida como um evento que consiste na relação entre o homem e o ambiente, dada uma concentração específica de poluente ambiental em um intervalo de tempo determinado.
A Organização Mundial de Saúde, em sua publicação denominada "Diretrizes de Saúde para Eventos de Fogo em Vegetação", esclarece que a queima de biomassa gera problemas basicamente em duas áreas ambientais ${ }^{5}$ :

- Poluição atmosférica - impacto direto da fumaça na saúde humana e na economia, influência de gases e emissões de partículas na composição da atmosfera.

- Biodiversidade - conseqüências deletérias no desempenho dos ecossistemas e na estabilidade da paisagem.

A maior parte da literatura que trata de emissões atmosféricas produzidas pela queima de biomassa aponta que estas causam efeitos deletérios à saúde humana. A Organização Mundial da Saúde sugere que há impacto sobre a mortalidade diária, admissões hospitalares, visitas à emergência e ao ambulatório e sobre a função pulmonar, como mostra a Figura $1^{6}$. Contudo, os estudos dos efeitos que o material particulado produzido pelas queimadas pode causar à saúde humana são escassos $^{3}$. Parte deste problema ocorre devido à variedade de aspectos que envolvem o problema, tornando difícil separar causas isoladas de um determinado efeito.

Com relação aos padrões de qualidade do ar para o Estado de São Paulo, o governo estadual publicou a Lei no 997 , de 31 de

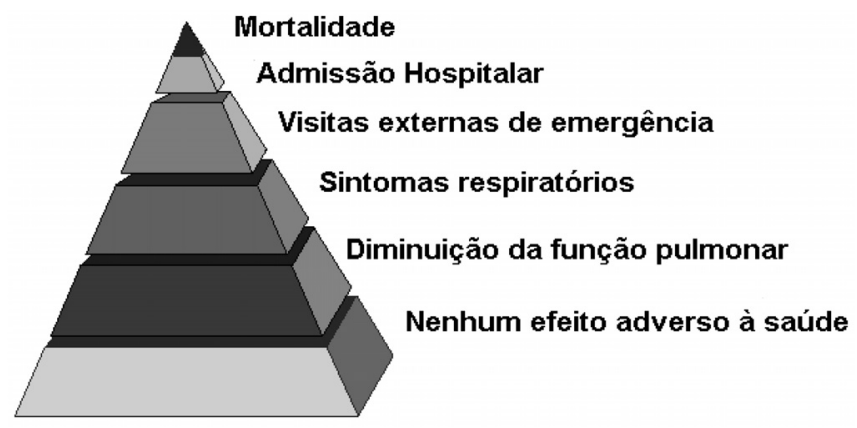

Figura 1 - Pirâmide representando a proporcionalidade populacional para cada situação possível em casos de exposição ao produto da queima de biomassa ${ }^{6}$.

Figure 1 - Piramid representing population ratios for each possible situation in cases of exposure to products resulting from biomass fire ${ }^{6}$. 
maio de 1976, que dispõe sobre a Prevenção e o Controle da Poluição do Meio Ambiente. Tal lei foi regulamentada pelo decreto estadual no 8.468 , de 8 de setembro de 1976. Especificamente no Título 3, Capítulo I, alguns padrões foram definidos para a qualidade do ar. Este decreto define um plano de emergência para episódios críticos de poluição do ar e, somente então coloca situações de proibição de queima, ainda que, de modo genérico.

Mais adiante, em 19 de setembro de 2002, o governo do Estado de São Paulo publicou a Lei no 11.241 que dispõe sobre a eliminação da queima da palha da canade-açúcar. Esta lei foi regulamentada pelo decreto no 47.700, de 11 de março de 2003, que apresenta em seu artigo $2^{\circ}$ a tabela de eliminação gradativa, no prazo de 30 anos (finalizando em 2031). Até lá, a população que vive no entorno das áreas de queimada continuará a sofrer as interferências negativas do "carvãozinho" no cotidiano.

Considerando que há uma relação entre a distribuição dos agravos à saúde e o espaço onde eles ocorrem, é importante conhecer como se dá tal distribuição, bem como a evolução dos impactos à saúde relativos a esta população. Da mesma forma que a epidemiologia e a cartografia sempre estiveram interrelacionadas, a construção de mapas contendo dados de saúde permite observar fenômenos que nem sempre são possíveis de identificar com dados dispostos em tabelas. A utilização de ferramentas de Sistemas de Informação Geográfica - SIG, apresenta-se como instrumental agregador e analisador de variáveis, necessário para estudos ecológicos desta natureza.

Os recursos computacionais permitem trabalhar com grandes volumes de dados de forma mais rápida e eficiente. As técnicas de geoprocessamento facilitam a associação de bases gráficas e não gráficas de forma a produzir cartas temáticas com mais qualidade e precisão. Com isso, os produtos gerados, através de convenções e representações, são convertidos em informações que podem subsidiar decisões e conseqüente desencadeamento de ações de saúde pública.

No conjunto de elementos apresentados, este trabalho se propõe a unir os aspectos de saúde pública aos fatores ambientais através da tecnologia da informação, especificamente um SIG (Sistema de Informação Geográfica) agregando dados sobre internações hospitalares aos dados sobre queimadas da palha de cana-de-açúcar, a fim de verificar a hipótese de uma correlação entre estas variáveis.

\section{Metodologia}

Para verificar uma possível correlação entre a incidência de doenças respiratórias crônicas e a exposição aos produtos da queima de palha de cana-de-açúcar, optouse por um estudo epidemiológico ecológico devido à abordagem que se pretendia.

É importante salientar que fatores como condições climáticas e padrão socioeconômico do local de estudo podem ser considerados fatores de confusão em pesquisas que envolvem comparação entre localidades.

A utilização de tecnologias de geoprocessamento possibilita identificar relações entre a distribuição de agravos à saúde e os condicionantes ambientais, agregando e mapeando variáveis de fontes distintas. $\mathrm{Na}$ presente pesquisa foram mapeados 15.986 focos de calor e 116.215 internações em 645 municípios do Estado de São Paulo, no período de janeiro de 2000 a dezembro de 2004.

Os dados sobre internações foram obtidos do sistema de informações hospitalares do SUS - SIH/SUS. São informações provenientes de hospitais da rede pública ou privada, conveniada do SUS, que enviam informações das internações por AIH (Autorização de Internação Hospitalar) para os gestores municipais e estaduais. As AIH são processadas pelo DATASUS e seus dados são disponibilizados para consulta pública na Internet .

Este trabalho foi estruturado na perspectiva de se obter resultados em duas 
abordagens escalares. Primeiramente construiu-se um SIG com abrangência regional, considerando os 645 municípios. O resultado desta fase indicou regiões candidatas à observação de incidência acima do padrão, em função das queimadas, que carecem de estudos mais aprofundados sobre os fenômenos abordados.

A segunda fase consistiu da construção de um SIG em escala mais detalhada, abrangendo a meso-região administrativa de Bauru, possibilitando estudar exposição e efeito em populações no entorno de áreas canavieiras.

Após a construção do SIG iniciou-se uma análise dos dados geo-referenciados que resultou nas cartas temáticas elaboradas neste estudo.

Todos os dados não gráficos utilizados neste estudo são secundários, gerados por instituições públicas e disponibilizados gratuitamente pela Internet, à exceção da carta digital da malha municipal do Estado de São Paulo que é comercializada pelo IBGE (Instituto Brasileiro de Geografia e Estatística) ${ }^{7}$. O mosaico de cobertura vegetal indicando áreas de plantio de canade-açúcar foi obtido junto ao Centro de Tecnologia Canavieira em Piracicaba ${ }^{8}$.

Os focos de calor são indícios de queimada e estão disponíveis na Internet, em formato de planilha eletrônica e foto de satélite, com informações a partir de $01 \mathrm{de}$ junho de $1998^{9}$. O conjunto de dados utilizado foi obtido pela Internet no site do DPI - Divisão de Processamento de Imagens do INPE (Instituto Nacional de Pesquisas Espaciais), que disponibiliza o banco de dados geo-referenciado dos focos de queimada detectados por satélite. Este estudo utilizou especificamente os dados do satélite NOAA 12 no período noturno, devido ao fato de as queimadas em cultura canavieira ocorrerem à noite, por força da legislação ambiental do Estado de São Paulo.

O conjunto de dados não gráfico foi convertido para o formato MS-Access 2000, com o intuito de facilitar as consultas e a integração com a ferramenta de SIG.

Os dados gráficos foram obtidos a par- tir da Malha Digital Municipal do Estado de São Paulo, compilada pelo IBGE - Instituto Brasileiro de Geografia e Estatística, na escala $1: 250.000$, que acompanha uma base de dados com informações censitárias dos municípios.

Os dados gráficos foram integrados aos demais através da ferramenta MapInfo, versão 6.0, que possibilitou a geração dos mapas temáticos obtidos no estudo.

Com relação à incidência, as taxas municipais foram obtidas a partir dos dados do Datasus (internação) e do IBGE (população), estabelecendo um padrão de comparação de incidência média trimestral para a região de Bauru. Em específico para os dados do Datasus, foram utilizados os filtros “Conteúdo=Internações” e “Grupo de procedimentos=Afecções das vias áreas superiores”. Este último agrega os procedimentos referentes às doenças descritas no Capítulo 10 - Doenças do aparelho respiratório da CID-10. Optou-se também por não especificar uma faixa etária.

\section{Resultados}

O Estado de São Paulo possui 15 mesoregiões administrativas. Algumas mesoregiões, como Ribeirão Preto, Araraquara e Piracicaba, possuem grandes extensões de monocultura canavieira.

Outras meso-regiões, como Araçatuba, Assis, Bauru, Presidente Prudente e São José do Rio Preto, situam-se próximas de pólos de produção sucro-alcooleira e boa parte de seus territórios é ocupada por cultura da cana-de-açúcar. Por outro lado, algumas meso-regiões, como Itapetininga, Litoral Sul Paulista, Marília, Metropolitana de São Paulo, Macro Metropolitana Paulista e Vale do Paraíba, pouco ou nada investem na cultura da gramínea.

Através da sobreposição de cartas temáticas das informações sobre áreas de plantio de cana de açúcar, limites de mesoregiões e de municípios e focos de calor, foi possível observar a coincidência das concentrações de tais focos sobre as áreas de plantio de cana-de-açúcar, comprovan- 
do que a utilização da técnica de queimada da palha de cana ainda é largamente utilizada no Estado. Este padrão foi observado nos mapas elaborados com dados dos anos 2000 a 2004. O Mapa 1 permite a visualização deste padrão.

Em seguida, ainda utilizando a técnica de sobreposição de cartas, foi possível observar que os municípios com os mais altos coeficientes de incidência de internações por afecções das vias respiratórias situam-se nas áreas onde ocorrem as maiores concentrações de queimadas.. A partir dos dados coletados, obteve-se o coeficiente de incidência para o Estado de São Paulo na ordem de 5,5 casos para 10.000 habitantes. Os municípios foram classificados com hachuras distintas em função da incidência abaixo ou acima do valor obtido para o Estado. Os mapas temáticos gerados nesta etapa permitiram observar o mesmo fenômeno nos 5 anos analisados (2000 a 2004). O Mapa 2 apresenta a distribuição de incidência das internações por afecções acumuladas para o ano de 2004.

É importante salientar que foram consideradas as internações por afecções das vias respiratórias registradas no SIH/SUS (Sistema de Informações Hospitalares do SUS $)^{11}$, que recebe dados de toda a rede pública, bem como da rede privada conveniada. Portanto, é provável haver sub-registro de dados, uma vez que a rede privada não notifica a totalidade dos casos para o SIH/SUS. No entanto, a rede pública atende a grande maioria das internações no Estado de São Paulo, e portanto também nas áreas que ocorrem as queimadas, minimizando problemas de sub-registro de internações nestas localidades.

Fator de confusão pode ocorrer na leitura dos dados, como outras fontes de queima, poluição de origem veicular e industrial, e condições atmosféricas. Por exemplo, o município de Ribeirão Preto apresentou incidência de internação por afecções elevada (entre 200 e 250 para cada 10.000 habitantes) no ano de 2004. Neste caso, a população ficou exposta aos compostos provenientes das queimadas ocorridas em toda a região, somados ao produto da poluição veicular, entre outras, acentuada na área urbana deste município.

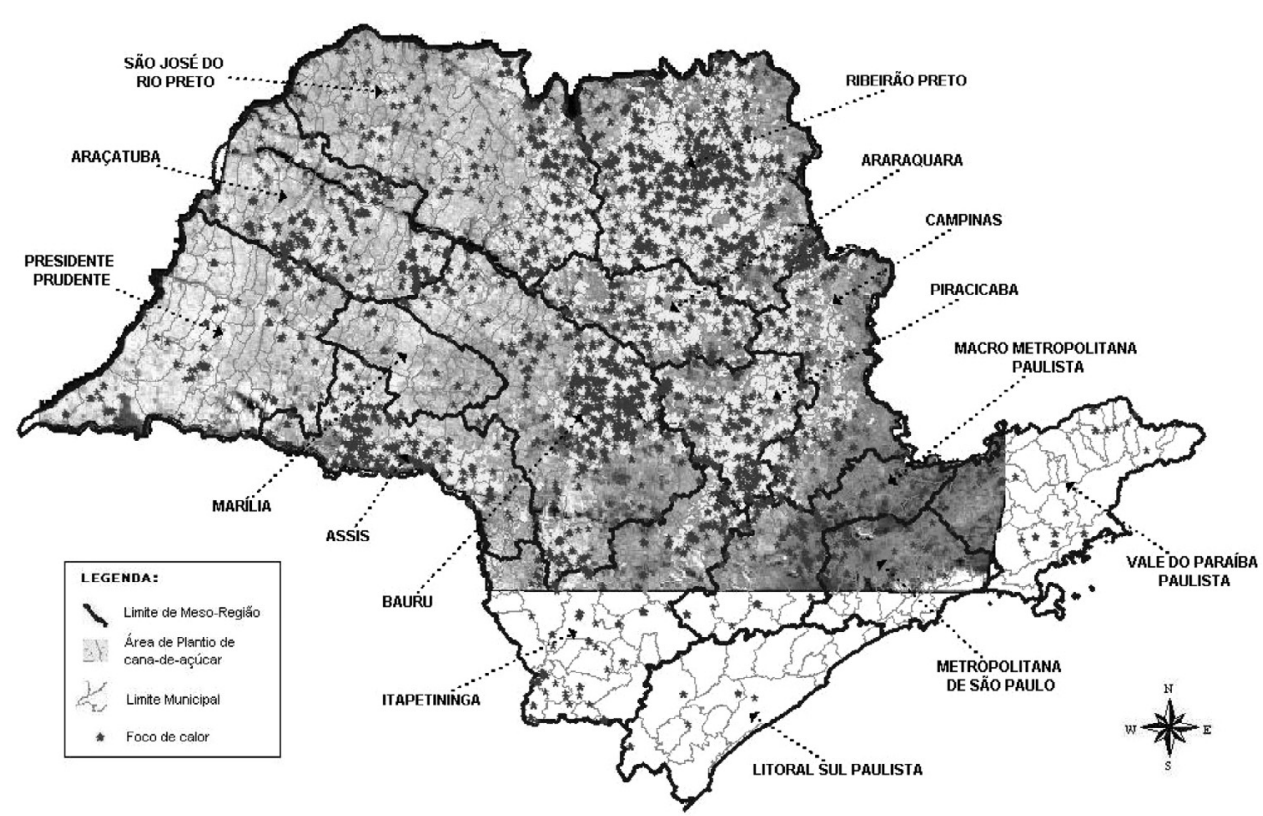

Mapa 1 - Focos de calor no estado de São Paulo, no ano de 2004, distribuídos nas meso-regiões administrativas estaduais ${ }^{10}$.

Map 1 - Heat Foci in the state of São Paulo, for the year 2004, distributed in state administrative meso-regions ${ }^{10}$. 


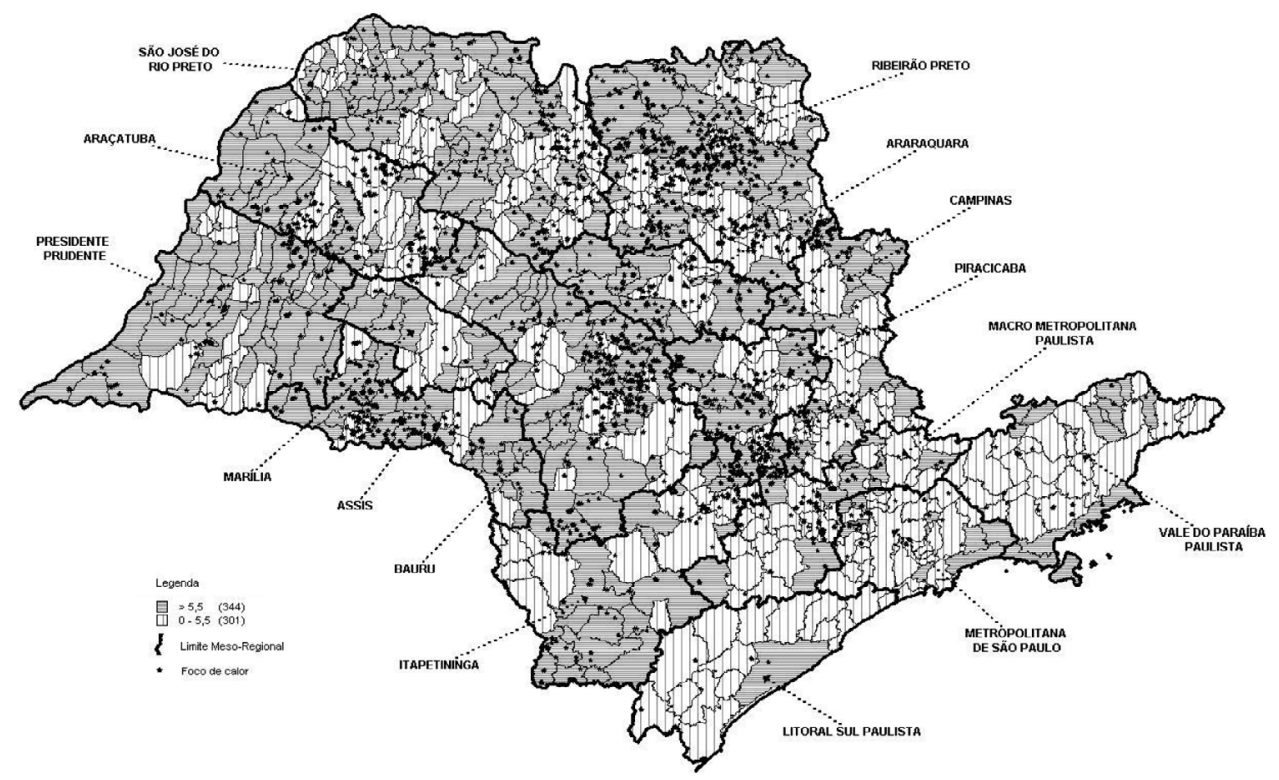

Mapa 2 - Distribuição da Incidência de internações por afecções nas vias respiratórias por 10.000 habitantes e focos de calor no estado de São Paulo, no ano de 2004, nas meso-regiões administrativas estaduais ${ }^{10}$.

Map 2 - Incidence of hospitalizations due to respiratory conditions per 10,000 inhabitants and heat foci in the state of São Paulo, for the year 2004, in state administrative meso-regions ${ }^{10}$.

A apresentação de dados, através de mapas, contribui no sentido de superar o problema de comparar eventos que não ocorrem necessariamente dentro das mesmas linhas divisórias estabelecidas pelo homem. Em outras palavras, os fenômenos ocorrem em espaços que a própria natureza determina, e não pela divisão política municipal ou meso-regional.

Os Mapas 3, 4, 5, 6 foram concebidos pela segmentação dos dados por trimestre. Esta construção possibilitou observar a evolução das variáveis no período de 1 ano. Cabe ressaltar que essa metodologia simplifica muito uma correta estimativa de áreas impactadas, uma vez que seria necessário elaborar uma modelagem de dispersão dos poluentes considerando variáveis como área queimada, umidade, velocidade e direção dos ventos, entre outras. No entanto, os atuais modelos gaussianos de pluma foram construídos considerando emissões em pontos específicos como as chaminés das indústrias. A modelagem de plumas para áreas difusas ainda carece da construção de modelos mais apropriados.
Nesta escala mais detalhada, os clusters (agrupamentos) de interesse para estudos ficam mais claros, permitindo uma melhor avaliação dos fenômenos pesquisados.

Os mapas temáticos construídos na fase anterior possibilitaram observar a distribuição correlacionada dos agravos ambientais e dos problemas de saúde. Em particular, a meso-região administrativa de Bauru despertou interesse devido ao fato de possibilitar agrupar seus 56 municípios em três situações distintas. A primeira, devido à grande concentração de áreas de plantio aglutinada em 17 municípios: Agudos, Areiópolis, Barra Bonita, Bariri, Bocaina, Boracéia, Borebi, Dois Córregos, Igaraçu do Tietê, Itajú, Itapuí, Jaú, Lençóis Paulista, Macatuba, Mineiros do Tietê, Pederneiras e São Manuel, pertencentes às micro-regiões de Jaú e Bauru. Este fato gerou intensa atividade de queimadas e causou vários transtornos à população que ali vive. Mais ao norte desta área, os municípios de Avaí, Cafelândia, Getulina, Guaiçara, Iacanga, Lins, Pirajuí, Promissão e Sabino, pertencentes às micro-regiões de Lins e Bauru, 


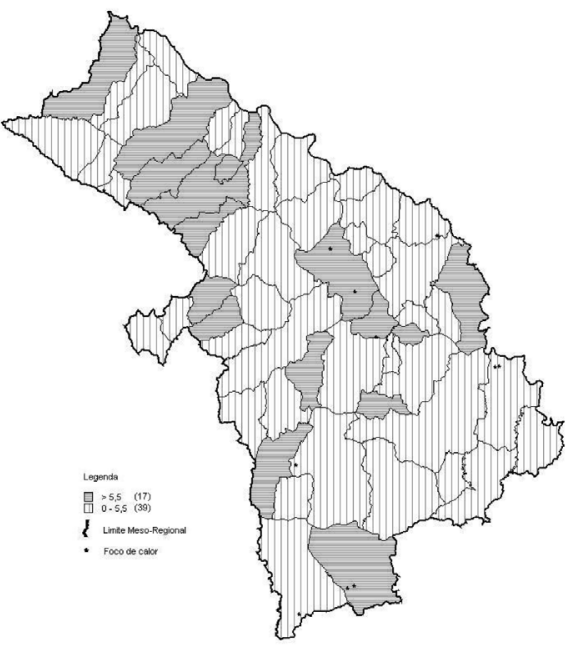

Mapa 3 - Distribuição da incidência de internações por afecções nas vias respiratórias por 10.000 habitantes e focos de calor na meso-região administrativa de Bauru, do estado de São Paulo no primeiro trimestre de 2004.

Map 3 - Incidence of hospitalizations due to respiratory conditions per 10,000 inhabitants and heat foci in the administrative meso-region of Bauru, state of São Paulo in the first quarter of 2004

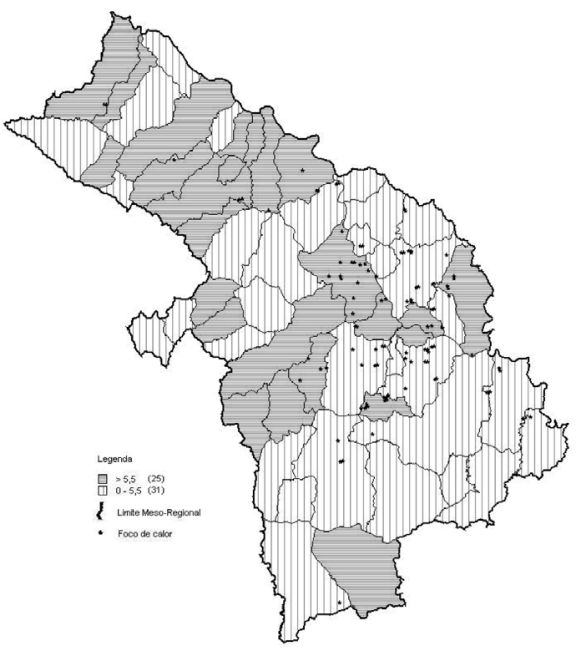

Mapa 4 - Distribuição da incidência de internações por afecções nas vias respiratórias por 10.000 habitantes e focos de calor na meso-região administrativa de Bauru, do estado de São Paulo no segundo trimestre de 2004.

Map 4 - Incidence of hospitalizations due to respiratory conditions per 10,000 inhabitants and heat foci in the administrative meso-region of Bauru, state of São Paulo in the second quarter of 2004.

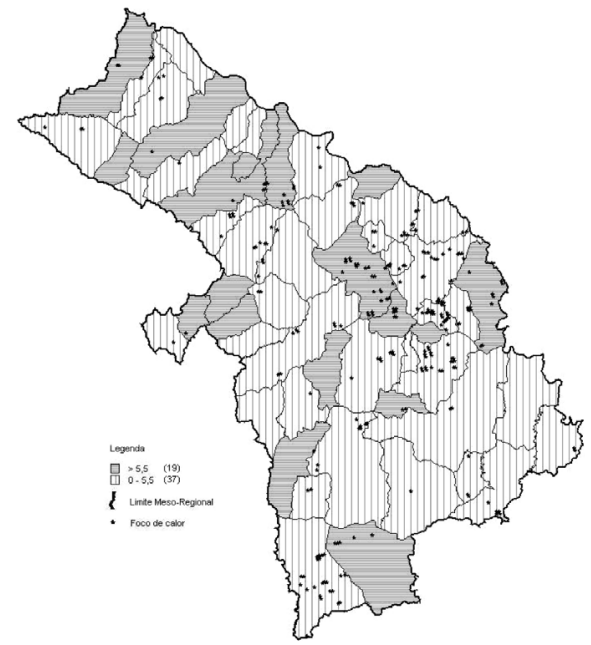

Mapa 5 - Distribuição da incidência de internações por afecções nas vias respiratórias por 10.000 habitantes e focos de calor na meso-região administrativa de Bauru, do estado de São Paulo no terceiro trimestre de 2004.

Map 5 - Incidence of hospitalizations due to respiratory conditions per 10,000 inhabitants and heat foci in the administrative meso-region of Bauru, state of São Paulo in the third quarter of 2004

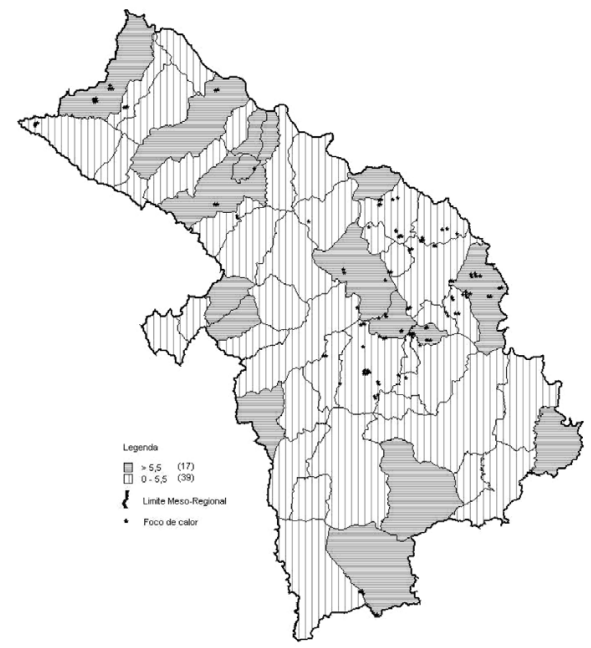

Mapa 6 - Distribuição da incidência de internações por afecções nas vias respiratórias por 10.000 habitantes e focos de calor na meso-região administrativa de Bauru, do estado de São Paulo no quarto trimestre de 2004.

Map 6 - Incidence of hospitalizations due to respiratory conditions per 10,000 inhabitants and heat foci in the administrative meso-region of Bauru, state of São Paulo in the fourth quarter of 2004. 
desenvolvem a cultura canavieira, porém com menor intensidade que o aglutinado anterior. Por fim, os demais municípios desta meso-região não apresentam as mesmas características, pois desenvolvem outros tipos de atividade econômica.

Os municípios de Pirajuí, Cafelândia e Duartina apresentaram as maiores incidências, com valores acima de 55 internações para cada 10.000 habitantes, 10 vezes maior que o padrão obtido para o Estado de São Paulo. A meso-região de Bauru apresentou um padrão de 14,05 internações para cada 10.000 habitantes no ano de 2004.

A micro-região de Jaú, composta por 12 dos 56 municípios da meso-região de Bauru, isoladamente é responsável por $45,8 \%$ dos focos de calor ocorridos em 2004 nesta meso-região, conforme apresenta a Tabela 1, e também totalizou $38,5 \%$ das internações por afecção das vias aéreas superiores neste mesmo ano.

Considerando que no ano de 2004 produziram-se 496 focos de calor no entorno de uma área que abriga 292.197 habitantes, proporcionalmente este processo gerou uma queimada para cada 500 habitantes. Outrossim, tal população representa $23,9 \%$ da meso-região e tem que conviver com $45,8 \%$ dos focos de calor e sobreviver com 38,5\% das internações, que provavelmente ocorreram em função do problema ambiental exposto.

A comparação dos valores, dispostos na tabela anterior, demonstra possível interrelação na micro-região de Jaú. Todos os municípios da micro-região de Jaú (Bariri, Barra Bonita, Bocaina, Boracéia, Dois Córregos, Igaraçu do Tietê, Itajú, Itapuí, Jaú, Macatuba, Mineiros do Tietê e Pederneiras) apresentam incidência maior que zero para o agravo. Juntos, compõem um núcleo de intenso cultivo de cana-de-açúcar e possuem vários focos de calor dentro de seus limites municipais. Porém, quando a comparação é feita para a mesoregião, a correlação não é tão linear.

Mais ao norte desta área, os municípios de Avaí, Cafelândia, Getulina, Guaiçara, Iacanga, Lins, Pirajuí, Promissão e Sabino, pertencentes às micro-regiões de Lins e Bauru, desenvolvem a cultura canavieira, porém com menor intensidade que o aglutinado anterior. Por fim, os demais municípios desta meso-região não apresentam as mesmas características, pois desenvolvem outros tipos de atividade econômica. A análise dos mapas permite verificar, por exemplo, que o município de Pirajuí apresenta áreas de plantio de canade-açúcar. Os mapas 3 a 6 evidenciam a presença de internações e queimadas neste mesmo município. Fato inverso ocorre no município de Duartina, os mesmos mapas mostram que a área deste município não produz cana-de-açúcar e não apresenta focos de calor. Porém, apresenta a mesma classificação de internações que Pirajuí. Logo, a correlação entre queima-

Tabela 1 - Comparativo de ocorrência de focos de calor e Internações por afecção das vias respiratórias na meso-região de Bauru e micro-região de Jaú no ano de 2004 9,11.

Table 1 - Comparison of heat foci and hospitalizations due to respiratory conditions in the meso-region of Bauru and microregion of Jaú in $2004^{9,11}$.

\begin{tabular}{lcccccc}
\hline Trimestres & \multicolumn{2}{c}{$\begin{array}{c}\text { Meso-região Bauru } \\
(1.220 .540\end{array}$} & \multicolumn{3}{c}{ habitantes) } \\
$\begin{array}{c}\text { Focos } \\
\text { de calor }\end{array}$ & Internações & Focos & Micro-regiãoJaú \\
de calor & (146.874 habitantes) \\
Internações & \% Forcos & \% Internações \\
de Calor & \\
\hline $1^{\circ}$ & 13 & 314 & 5 & 122 & 38,5 & 38,8 \\
$2^{\circ}$ & 104 & 522 & 46 & 180 & 44,2 & 34,5 \\
$3^{\circ}$ & 257 & 459 & 110 & 203 & 42,8 & 44,2 \\
$4^{\circ}$ & 122 & 420 & 66 & 155 & 54,1 & 36,9 \\
Total & 496 & 1715 & 227 & 660 & 45,8 & 38,5 \\
\hline
\end{tabular}


da e internação por afecções não permite explicar da mesma forma a incidência nos dois municípios.

Fato diferenciado ocorre na micro-região de Jaú, onde mapas e gráficos apontam uma possível correlação queimadas/ internações, mas estes resultados não são determinísticos, apenas sugerem hipóteses para estudos mais aprofundados.

\section{Considerações Finais}

Neste ano de 2005, mais uma vez, como ocorreu nos últimos cinco anos, outra safra de cana-de-açúcar aconteceu no Estado de São Paulo. Grandes áreas de monocultura canavieira foram queimadas e proporcionaram subempregos a um milhão de trabalhadores que vivem do corte da cana. Segundo o decreto 47.700, de 11 de março de 2003, ainda será assim por mais 26 anos até o prazo final de eliminação do processo de queimada em 2031.

Ainda que não houvesse correlação encontrada neste estudo para justificar os agravos à saúde, só os transtornos vivenciados pela população impactada, tais como sujeira em casas e locais públicos, acidentes em rodovias por falta de visibilidade e interrupção de fornecimento de energia elétrica por problemas nas linhas de transmissão, já seria suficiente para eliminar as queimadas. Soma-se a isso os condicionantes ambientais envolvidos na questão, queima de biomassa, emissão de $\mathrm{CO}_{2}$, entre outros compostos, gerando impactos na natureza desconhecidos ainda pelo homem.

Considerando que a saúde pública busca a promoção da saúde e a garantia da qualidade de vida das coletividades, este trabalho buscou apresentar ferramentas fomentadoras de hipóteses para estudos que vão ao encontro destes objetivos.

O aprofundamento destes estudos é importante para produzir evidências sólidas de modo a subsidiar a formulação e comprovação de hipóteses. Tal argumento se vale para criar mecanismos mais eficientes para auxiliar a tomada de decisão no âmbito das políticas públicas, embasando de forma mais adequada a legislação nesta agenda e as ações de vigilância ambiental.

Sabe-se das dificuldades comuns aos profissionais de saúde quanto ao uso de sofisticadas tecnologias computacionais em estudos desta natureza. Contudo, cabe ao profissional de informática ser um facilitador de recursos, articulando-se aos profissionais de saúde. Os SIGs estão presentes em muitos estudos de saúde pública, porém quase sempre estão limitados a um usuário que se interessou pelos recursos e resolveu aprender mais sobre de que modo utilizá-lo.

Os mapas, em escala local, elaborados neste estudo apontaram possível correlação entre queima de cana-de-açúcar e internações por afecções respiratórias na micro-região de Jaú. Os resultados agregam mais riqueza de detalhes nesta escala, porém a natureza desta pesquisa não permitiu resultados determinísticos, somente dados para auxiliar a formulação de novas hipóteses. Assim sendo, este estudo estimula a elaboração de pesquisas mais aprofundadas para o tema em questão.

Conclui-se, portanto, que as tecnologias de SIG mostraram-se úteis neste estudo exploratório, pois possibilitaram observar, na mesma perspectiva, dados sobre a exposição humana aos compostos produzidos em queimadas e os problemas de saúde respiratória que impactaram a população.

\section{Referências}

1. EMBRAPA - Empresa Brasileira de Pesquisa Agropecuária. Agroecologia da cana-de-açúcar. Disponível em <http:/ /www.cana.cnpm.embrapa. br/ agroeco.html> Acessado em 8 de janeiro de 2004.
2. MRE - Ministério das Relações Exteriores. Disponível em <URL:http://www.mre.gov.br/cdbrasil/itamaraty/web/ port/economia/agroind/apresent/apresent.htm.> Acessado em 5 de abril de 2006. 
3. Zancul A. O efeito da queimada de cana-de-açúcar na qualidade do ar de região de Araraquara. São Carlos [dissertação de mestrado]. São Carlos: Escola de Engenharia de São Carlos da USP; 1998.

4. Castro HA, Gouveia N, Escamilla-Cejudo JA. Questões metodológicas para a investigação dos efeitos da poluição do ar na saúde. Rev Bras Epidemiol 2003; 6(2): $135-49$.

5. WHO - World Health Organization. Health Guidelines for Vegetation Fire Events. ed. Schwela DH, Goldammer JG, Morawska LH, Simpson O. Genebra: World Health Organization; 1999. Disponível em <URL:http:/ / www.who.int/docstore/peh/Vegetation_fires/ Backgroundpapers/ BackgrPapl.pdf $>$ Acessado em 6 de janeiro de 2004.

6. WHO - World Health Organization. Guias para la Calidad del Aire. Genebra: World Health Organization; 1999.

7. IBGE - Instituto Brasileiro de Geografia e Estatística. Catálogo IBGE. Disponível em <URL: http:/ / www.ibge.gov.br> Acessado em 8 de janeiro de 2004.
8. CTC. Mosaico digital obtido por satélite das áreas de cultivo de cana-de-açúcar no Estado de São Paulo. Centro de Tecnologia Canavieira. São Paulo; 2005.

9. INPE - Instituto Nacional de Pesquisas Espaciais. Monitoramento de Queimadas. [Banco de dados sobre queimadas]. Disponível em <URL: http://www. dpi.inpe.br/ proarco/bdqueimadas > Acessado em 24 de março de 2005.

10. Lopes FS. A Utilização de Sistemas de Informação Geográfica no estudo da exposição humana aos produtos da queima da palha de cana-de-açúcar (saccharum $s p$ ) no Estado de São Paulo [dissertação de mestrado]. São Paulo: Faculdade de Saúde Pública da Universidade de São Paulo; ano?.

11. DATASUS - Processamento de Dados do SUS. Procedimentos hospitalares do SUS. Disponível em <http://tabnet.datasus. gov.br/cgi/deftohtm.exe?sih/ cnv/prsp.def $>$ Acessado em 8 de janeiro de 2004.

Recebido em: 20/01/06

Versão reformulada reapresentada em: 18/04/06 Aprovado em: 30/05/06 